\title{
New Stability Criterion for Takagi-Sugeno Fuzzy Cohen-Grossberg Neural Networks with Probabilistic Time-Varying Delays
}

\author{
Xiongrui Wang, ${ }^{1}$ Ruofeng Rao, ${ }^{2}$ and Shouming Zhong ${ }^{3}$ \\ ${ }^{1}$ Department of Mathematics, Yibin University, Yibin 644000, China \\ ${ }^{2}$ Department of Mathematics, Chengdu Normal University, Chengdu, Sichuan 611130, China \\ ${ }^{3}$ College of Mathematics, University of Electronic Science and Technology of China, Chengdu 611731, China \\ Correspondence should be addressed to Ruofeng Rao; ruofengrao@163.com
}

Received 2 July 2017; Revised 10 October 2017; Accepted 24 October 2017; Published 16 November 2017

Academic Editor: Renming Yang

Copyright (C) 2017 Xiongrui Wang et al. This is an open access article distributed under the Creative Commons Attribution License, which permits unrestricted use, distribution, and reproduction in any medium, provided the original work is properly cited.

\begin{abstract}
A new global asymptotic stability criterion of Takagi-Sugeno fuzzy Cohen-Grossberg neural networks with probabilistic timevarying delays was derived, in which the diffusion item can play its role. Owing to deleting the boundedness conditions on amplification functions, the main result is a novelty to some extent. Besides, there is another novelty in methods, for LyapunovKrasovskii functional is the positive definite form of $p$ powers, which is different from those of existing literature. Moreover, a numerical example illustrates the effectiveness of the proposed methods.
\end{abstract}

\section{Introduction}

Cohen-Grossberg neural networks (CGNNs) have many practical applications, like artificial intelligence, parallel computing, image processing and recovery, and so on ([1-6]). But the success of these applications largely depends on whether the system has some stability, and so people began to be interested in the stability analysis of the system. In recent decades, reaction-diffusion neural networks have received much attention ([7-13]), including various Laplacian diffusion ([6, 14-20]). Besides, people are paying more and more attention to fuzzy neural network system ([21-34]), due to encountering always some inconveniences such as the complicity, the uncertainty, and vagueness ([27, 35-37]). For example, in [27], Zhu and Li investigated the following fuzzy CGNNs model:

$$
\begin{gathered}
d x_{i}(t)=\left\{-a_{i}\left(x_{i}(t)\right)\left[b_{i}\left(x_{i}(t)\right)-\bigwedge_{j=1}^{n} a_{i j} f_{j}\left(x_{j}(t)\right)\right.\right. \\
-\bigvee_{j=1}^{n} b_{i j} g_{j}\left(x_{j}(t)\right)-\bigwedge_{j=1}^{n} c_{i j} f_{j}\left(x_{j}(t-\tau)\right)
\end{gathered}
$$

$$
\begin{aligned}
& \left.\left.-\bigvee_{j=1}^{n} d_{i j} g_{j}\left(x_{j}(t-\tau)\right)\right]\right\} d t \\
& +\sum_{j=1}^{n} \sigma_{i j}\left(x_{j}(t), x_{j}(t-\tau)\right) d w_{j}(t), \\
& x_{i}(t)=\phi_{i}(t),-\tau \leqslant t \leqslant 0 .
\end{aligned}
$$

In [36], Muralisankar and Gopalakrishnan studied the following T-S fuzzy neutral type CGNNs with distributed delays:

$$
\begin{aligned}
& d x_{i}(t)=\sum_{j=1}^{r} h_{j}(\omega(t))\left\{-A_{j}(x(t))\left[B_{j}(x(t))\right.\right. \\
& -C_{j} f(x(t-\tau(t)))-M_{j} \int_{t-\rho(t)}^{t} f(x(s)) d s \\
& \left.\left.\quad-D_{j} \dot{x}(t-r(t))\right]\right\} .
\end{aligned}
$$


Besides, Balasubramaniam and Syed Ali discussed TakagiSugeno fuzzy Cohen-Grossberg BAM neural networks with discrete and distributed time-varying delays in [37].

Note that there is the following bounded condition on amplification functions in many literatures (see, e.g., [38, Theorem 4]) related to CGNNs:

$$
0<\underline{a}_{i} \leqslant a_{i}(r) \leqslant \bar{a}_{i}, \quad r \in R, i=1,2, \ldots, n .
$$

So, in this paper, we try to delete this bounded condition on amplification functions. This is the main purpose of this paper.

\section{Preliminaries}

Consider the following fuzzy Takagi-Sugeno $p$-Laplace partial differential equations with distributed delay.

Fuzzy Rule $j$. IF $\omega_{1}(t)$ is $\mu_{j 1}$ and $\cdots \omega_{s}(t)$ is $\mu_{j s}$ THEN

$$
\begin{aligned}
& \frac{\partial u}{\partial t}=\nabla \cdot\left(\mathscr{D}(t, x, u) \circ \nabla_{p} u\right)-A(u)[B(u) \\
& \left.\quad-C_{j} f(u(t-\tau(t), x))-M_{j} \int_{t-\rho(t)}^{t} f(u(s, x)) d s\right], \\
& u(\theta, x)=\phi(\theta, x), \quad(\theta, x) \in(-\infty, 0] \times \Omega, \\
& u(t, x)=0 \in R^{n}, \quad(t, x) \in R \times \partial \Omega,
\end{aligned}
$$

where $\Omega$ is an arbitrary open bounded subset in $R^{m}$. $\omega_{k}(t)(k=1,2, \ldots, s)$ is the premise variable and $\mu_{j k}(j=$ $1,2, \ldots, r ; k=1,2, \ldots, s)$ is the fuzzy set that is characterized by membership function. And $r$ is the number of the IF-THEN rules; $s$ is the number of the premise variables. $u(t, x)=\left(u_{1}(t, x), u_{2}(t, x), \ldots, u_{n}(t, x)\right)^{T} \in R^{n}$, where $u_{i}(t, x)$ is the state variable of the $i$ th neuron and the $j$ th neuron at time $t$ and in space variable $x$. Matrix $\mathscr{D}(t, x, u)=\left(\mathscr{D}_{i j}(t, x, u)\right)_{n \times m}$ with each $\mathscr{D}_{i j}(t, x, u) \geqslant 0$, and $\mathscr{D}_{i j}(t, x, u)$ is diffusion operator. $\mathscr{D}(t, x, u) \circ \nabla_{p} u=$ $\left(\mathscr{D}_{j k}(t, x, u)\right)\left|\nabla u_{i}\right|^{p-2}\left(\partial u_{i} / \partial x_{k}\right)_{n \times m}$ denotes the Hadamard product of matrix $\mathscr{D}(t, x, u)$ and $\nabla_{p} u$ (see [39] for details). Matrices $A(u)=\operatorname{diag}\left(a_{1}\left(u_{1}\right), a_{2}\left(u_{2}\right), \ldots, a_{n}\left(u_{n}\right)\right)$ and $B(u)=$ $\operatorname{diag}\left(b_{1}\left(u_{1}\right), b_{2}\left(u_{2}\right), \ldots, b_{n}\left(u_{n}\right)\right)$, where $a_{i}\left(u_{i}\right)$ and $b_{i}\left(u_{i}\right)$ represent an amplification function at time $t$ and an appropriate behavior function at time t. $C_{j}=\left(c_{i k}^{(j)}\right)_{n \times n}$ is the connection matrix. Time delays $\tau(t) \in[0,+\infty) . f(u(t-\tau(t), x))=$ $\left(f_{1}\left(u_{1}(t-\tau(t) t, x)\right), f_{2}\left(u_{2}(t-\tau(t), x)\right), \ldots, f_{n}\left(u_{n}(t-\tau(t), x)\right)\right)^{T}$ is the activation function of the neurons. And the second and third equations of (4) imply the initial condition and the Dirichlet boundary condition, respectively.

By way of a standard fuzzy inference method, (4) can be inferred as follows.

$$
\begin{aligned}
\frac{\partial u}{\partial t} & =\nabla \cdot\left(\mathscr{D}(t, x, u) \circ \nabla_{p} u\right)-A(u(t, x))[B(u(t, x)) \\
& -\sum_{j=1}^{r} h_{j}(\omega(t))\left(C_{j} f(u(t-\tau(t), x))\right.
\end{aligned}
$$

$$
\begin{aligned}
& \left.\left.+M_{j} \int_{t-\rho(t)}^{t} f(u(s, x)) d s\right)\right], \\
& u(\theta, x)=\phi(\theta, x), \quad(\theta, x) \in(-\infty, 0] \times \Omega, \\
& u(t, x)=0 \in R^{n}, \quad(t, x) \in R \times \partial \Omega,
\end{aligned}
$$

where $\omega(t)=\left[\omega_{1}(t), \omega_{2}(t), \ldots, \omega_{s}(t)\right]^{T}$ and $h_{j}(\omega(t))=$ $w_{j}(\omega(t)) / \sum_{k=1}^{r} w_{k}(\omega(t)), w_{j}(\omega(t)): R^{s} \rightarrow[0,1](j=$ $1,2, \ldots, r)$ is the membership function of the system with respect to the fuzzy rule $j . h_{j}$ can be regarded as the normalized weight of each IF-THEN rule, satisfying $h_{j}(\omega(t)) \geqslant 0$ and $\sum_{j=1}^{r} h_{j}(\omega(t))=1$.

Next, we consider the following information for probability distribution of time delays $\tau(t)$ :

$$
\begin{gathered}
\mathbb{P}\left(0 \leqslant \tau(t) \leqslant \tau_{1}\right)=c_{0}, \\
\mathbb{P}\left(\tau_{1}<\tau(t) \leqslant \tau_{2}\right)=1-c_{0} .
\end{gathered}
$$

Here the nonnegative scalar $c_{0} \leqslant 1$. Define a random variable as follows:

$$
\mathfrak{S}(t)= \begin{cases}1, & 0 \leqslant \tau(t) \leqslant \tau_{1} \\ 0, & \tau_{1}<\tau(t) \leqslant \tau_{2} .\end{cases}
$$

So, in this paper, we consider the following Takagi-Sugeno (T-S) fuzzy system with probabilistic time-varying delays:

$$
\begin{aligned}
& \frac{\partial u}{\partial t}=\nabla \cdot\left(\mathscr{D}(t, x, u) \circ \nabla_{p} u\right)-A(u(t, x))\{B(u(t, x)) \\
& \quad-\sum_{j=1}^{r} h_{j}(\omega(t))\left[c_{0} C_{j} f\left(u\left(t-\tau_{1}(t), x\right)\right)+\left(1-c_{0}\right)\right. \\
& \cdot C_{j} f\left(u\left(t-\tau_{2}(t), x\right)\right)+\left(\mathfrak{c}-c_{0}\right) \\
& \quad \cdot\left(C_{j} f\left(u\left(t-\tau_{1}(t), x\right)\right)-C_{j} f\left(u\left(t-\tau_{2}(t), x\right)\right)\right) \\
& \left.\left.\quad+M_{j} \int_{t-\rho(t)}^{t} f(u(s, x)) d s\right]\right\} \\
& u(\theta, x)=\phi(\theta, x), \quad(\theta, x) \in(-\infty, 0] \times \Omega, \\
& u(t, x)=0 \in R^{n}, \quad(t, x) \in R \times \partial \Omega .
\end{aligned}
$$


System (8) includes the following integrodifferential equations:

$$
\begin{aligned}
& \frac{d x(t)}{d t}=-A(x(t))\left\{B(x(t))-\sum_{j=1}^{r} h_{j}(\omega(t))\right. \\
& \cdot\left[c_{0} C_{j} f\left(x\left(t-\tau_{1}(t)\right)\right)+\left(1-c_{0}\right)\right. \\
& \cdot C_{j} f\left(x\left(t-\tau_{2}(t)\right)\right)+\left(\mathfrak{C}-c_{0}\right) \\
& \cdot\left(C_{j} f\left(x\left(t-\tau_{1}(t)\right)\right)-C_{j} f\left(x\left(t-\tau_{2}(t)\right)\right)\right) \\
& \left.\left.+M_{j} \int_{t-\rho(t)}^{t} f(x(s)) d s\right]\right\}, \quad t \geqslant 0, \\
& x(\theta)=\phi(\theta), \quad \theta \in(-\infty, 0] .
\end{aligned}
$$

Particularly when $p=2$, system (8) degenerates into the so-called reaction-diffusion CGNNs:

$$
\begin{aligned}
& \frac{\partial u}{\partial t}=\nabla \cdot(\mathscr{D}(t, x, u) \circ \nabla u)-A(u(t, x))\{B(u(t, x)) \\
& -\sum_{j=1}^{r} h_{j}(\omega(t))\left[c_{0} C_{j} f\left(u\left(t-\tau_{1}(t), x\right)\right)+\left(1-c_{0}\right)\right. \\
& \cdot C_{j} f\left(u\left(t-\tau_{2}(t), x\right)\right)+\left(\mathfrak{C}-c_{0}\right) \\
& \cdot\left(C_{j} f\left(u\left(t-\tau_{1}(t), x\right)\right)-C_{j} f\left(u\left(t-\tau_{2}(t), x\right)\right)\right) \\
& \left.\left.+M_{j} \int_{t-\rho(t)}^{t} f(u(s, x)) d s\right]\right\}, \\
& u(\theta, x)=\phi(\theta, x), \quad(\theta, x) \in(-\infty, 0] \times \Omega, \\
& u(t, x)=0 \in R^{n}, \quad(t, x) \in R \times \partial \Omega .
\end{aligned}
$$

Throughout this paper, we assume $p=p_{1} / p_{2}>1$ with $p_{1}$ being even number and $p_{2}$ being odd number. Besides, suppose that the following conditions hold:

(H1) There exist positive definite matrices $\underline{A}=\operatorname{diag}\left(\underline{a}_{1}\right.$, $\left.\underline{a}_{2}, \ldots, \underline{a}_{n}\right)$ and $\bar{A}=\operatorname{diag}\left(\bar{a}_{1}, \bar{a}_{2}, \ldots, \bar{a}_{n}\right)$ such that

$$
0<\underline{a}_{i} \leqslant \frac{a_{i}(s)}{s^{p-2}} \leqslant \bar{a}_{i}, \quad 0 \neq s \in R, i=1,2, \ldots, n,
$$

where $A(u)=\operatorname{diag}\left(a_{1}\left(u_{1}\right), a_{2}\left(u_{2}\right), \ldots, a_{n}\left(u_{n}\right)\right)$ and $u=\left(u_{1}, u_{2}, \ldots, u_{n}\right)^{T} \in R^{n}$.

$(\mathrm{H} 2)$ There exists a positive definite matrix $\mathbb{B}=$ $\operatorname{diag}\left(b_{1}, b_{2}, \ldots, b_{n}\right)$ such that $b_{i}(0)=0$ and

$$
\frac{b_{i}(s)}{s} \geqslant b_{i}, \quad 0 \neq s \in R, i=1,2, \ldots, n .
$$

(H3) There is a positive definite matrix $F=\operatorname{diag}\left(F_{1}, F_{2}\right.$, $\ldots, F_{n}$ ) such that

$$
\left|f_{i}(s)\right| \leqslant F_{i}|s|, \quad s \in R, i=1,2, \ldots, n .
$$

From $(\mathrm{H} 1)-(\mathrm{H} 3)$, we know that $b_{i}(0)=f_{i}(0)=0$ and $u=0$ is an equilibrium of fuzzy system (8).

Remark 1 . There are numerous functions satisfying (H1). For example, if $p=4 / 3$, we may set

$$
a_{i}(s)=\frac{0.1\left(1+e^{-s^{2}}\right)}{\sqrt[3]{s^{2}}}, \quad \forall 0 \neq s \in R, a_{i}(0)=0.2 .
$$

It is obvious that

$$
\lim _{s \rightarrow 0} a_{i}(s)=\lim _{s \rightarrow 0} \frac{0.1\left(1+e^{-s^{2}}\right)}{\sqrt[3]{s^{2}}}=+\infty .
$$

So the function $a_{i}(s)$ is unbounded for $s \in R$. Moreover,

$$
0.1 \leqslant \frac{a_{i}(s)}{s^{p-2}}=0.1\left(1+e^{-s^{2}}\right) \leqslant 0.2 .
$$

One can know from (16) that $0.1 \leqslant a_{i}(s) / s^{p-2} \leqslant 0.2$ with $\underline{a}_{i}=0.1$ and $\bar{a}_{i}=0.2$.

Remark 2. The amplification function $a_{i}(s)$ defined as (7) is actually unbounded for $s \in R$. However, various bounded conditions always imposed restrictions on the amplification functions of existing literature $([3-6,9,10,24,27,28])$. Hence, our condition (H1) is weaker, which will make a corollary with regard to ordinary integrodifferential equations (9) become novel.

For convenience's sake, we need to introduce the following standard notations similarly as [38]:

$$
\begin{gathered}
Q=\left(q_{i j}\right)_{n \times n}>0(<0), \\
Q=\left(q_{i j}\right)_{n \times n} \geqslant 0(\leqslant 0), \\
Q_{1} \geqslant Q_{2}\left(Q_{1} \leqslant Q_{2}\right), \\
Q_{1}>Q_{2}\left(Q_{1}<Q_{2}\right), \\
\lambda_{\max }(\Phi), \\
\lambda_{\min }(\Phi), \\
|C|=\left(\left|c_{i j}\right|\right)_{n \times n}, \\
|u(t, x)|,
\end{gathered}
$$

and the identity matrix with compatible $I$.

(i) The Sobolev space $=\left\{u \in L^{p}: \mathscr{D} u \in L^{p}\right\}$ (see [40] for details). 
(ii) Denote by $\lambda_{1}$ the lowest positive eigenvalue of the boundary value problem (see [40] for details).

$$
\begin{aligned}
-\Delta_{p} \varphi(t, x) & =\lambda \varphi(t, x), \quad x \in \Omega \\
\varphi(t, x) & =0, \quad x \in \partial \Omega .
\end{aligned}
$$

Lemma 3. One has

$$
a^{q-1} b \leqslant \frac{q-1}{q} a^{q}+\frac{b^{q}}{q}, \quad \forall a, b \in(0,+\infty), q>1 .
$$

Note that Lemma 3 is the particular case of the famous Young inequality.

\section{Results and Discussion}

Lemma 4. Let $P=\operatorname{diag}\left(p_{1}, p_{2}, \ldots, p_{n}\right)$ be a positive definite matrix and $u$ be a solution of the fuzzy system (8). Then one has

$$
\int_{\Omega} u^{T} P\left(\nabla \cdot\left(D(t, x, u) \circ \nabla_{p} u\right)\right) d x \leqslant-\lambda_{1} \underline{p} D\|u\|_{p}^{p},
$$

where $D=\min _{i k}\left(\inf _{t, x, u} \mid D_{i j}(t, x, u)\right),\|u\|_{p}^{p}=\sum_{i=1}^{n} \int_{\Omega}\left|u_{i}\right|^{p} d x$, and $p$ is a positive scalar, satisfying $P>p I$.

Proof. Since $u$ is a solution of system (8), it follows by Gauss formula and the Dirichlet zero-boundary condition that

$$
\begin{gathered}
\int_{\Omega} \sum_{j=1}^{n} p_{j} u_{j} \sum_{k=1}^{m} \frac{\partial}{\partial x_{k}}\left(D_{j k}\left|\nabla u_{j}\right|^{p-2} \frac{\partial u_{j}}{\partial x_{k}}\right) d x \\
=-\sum_{k=1}^{m} \sum_{j=1}^{n} \int_{\Omega} p_{j} D_{j k}\left|\nabla u_{j}\right|^{p-2}\left(\frac{\partial u_{j}}{\partial x_{k}}\right)^{2} d x \\
\leqslant-\lambda_{1} D \underline{p} \sum_{j=1}^{n} \int_{\Omega}\left|u_{j}\right|^{p} d x=-\lambda_{1} \underline{p} D\|u\|_{p}^{p} .
\end{gathered}
$$

Remark 5. Lemma 4 extends the conclusion of [2, Lemma 2.1] and [10, Lemma 2.4] from Hilbert space $H_{0}^{1}(\Omega)$ to Banach space $W_{0}^{1, p}(\Omega)$. Particularly, in the case of $\Omega=(0, T) \subset R^{1}$ or $W_{0}^{1, p}(0, T)$, the first eigenvalue $\lambda_{1}=\left((2 / T) \int_{0}^{(p-1)^{1 / p}}(d t /(1-\right.$ $\left.\left.\left.t^{p} /(p-1)\right)^{1 / p}\right)\right)^{p}$ (see, e.g., [40]).

Theorem 6. If there exists a positive definite matrix $P=$ $\operatorname{diag}\left(p_{1}, p_{2}, \ldots, p_{n}\right)$ and two positive scalars $p, \bar{p}$ such that the following inequalities hold:

$$
\begin{aligned}
\lambda_{1} D \underline{p} & +\underline{p} \lambda_{\text {min }}(\underline{A} \mathbb{B})>\frac{n \bar{p}}{p} \sum_{j=1}^{r}\left((p-1)\left|c_{j}\right|\right. \\
& \left.+\rho(p-1)\left|m_{j}\right|+\frac{c_{0}\left|c_{j}\right|}{1-\tau_{1}}+\frac{\left(1-c_{0}\right)\left|c_{j}\right|}{1-\tau_{2}}+\rho\left|m_{j}\right|\right) \\
& \cdot \lambda_{\text {max }} \bar{A} \lambda_{\text {max }} F \\
P & >\underline{p} I, \\
P & <\bar{p} I,
\end{aligned}
$$

then the null solution of fuzzy system (8) is globally asymptotically stable, where matrices $C_{j}=\left(c_{i k}^{(j)}\right)_{n \times n}, M_{j}=\left(m_{i k}^{(j)}\right)_{n \times n}$, $\left|c_{j}\right|=\max _{i k}\left|c_{i k}^{(j)}\right|,\left|m_{j}\right|=\max _{i k}\left|m_{i k}^{(j)}\right|$, and $\tau_{1}^{\prime}(t) \leqslant \tau_{1}<$ $1, \tau_{2}^{\prime}(t) \leqslant \tau_{2}<1,0 \leqslant \rho(t) \leqslant \rho$.

Proof. Firstly, we can conclude from (H1)-(H3) that $u=0$ is an equilibrium point for system (8).

Next, consider the Lyapunov-Krasovskii functional:

$$
V(t)=V_{1}(t)+\frac{1}{1-\tau_{1}} V_{2}(t)+\frac{1}{1-\tau_{2}} V_{3}(t)+V_{4}(t),
$$

where

$$
\begin{aligned}
& V_{1}(t)=\int_{\Omega} u^{T}(t, x) P u(t, x) d x=\sum_{i=1}^{n} \int_{\Omega} p_{i} u_{i}^{2} d x, \\
& V_{2}(t)=2 c_{0} n \frac{\bar{p}}{p}\left(\sum_{j=1}^{r}\left|c_{j}\right|\right) \\
& \cdot \lambda_{\max } \bar{A} \lambda_{\max } F \sum_{k=1}^{n} \int_{t-\tau_{1}(\mathrm{t})}^{t} \int_{\Omega}\left|u_{k}(s, x)\right|^{p} d x d s, \\
& V_{3}(t)=2\left(1-c_{0}\right) n \frac{\bar{p}}{p}\left(\sum_{j=1}^{r}\left|c_{j}\right|\right) \\
& \cdot \lambda_{\max } \bar{A} \lambda_{\max } F \sum_{k=1}^{n} \int_{t-\tau_{2}(t)}^{t} \int_{\Omega}\left|u_{k}(s, x)\right|^{p} d x d s, \\
& V_{4}(t)=2 \frac{\bar{p}}{p}\left(\sum_{j=1}^{r}\left|m_{j}\right|\right) \\
& \cdot \lambda_{\max } \bar{A} \lambda_{\max } F \sum_{i=1}^{n} \sum_{k=1}^{n} \int_{\Omega}\left(\int_{-\rho}^{0} d \zeta \int_{t+\zeta}^{t}\left|u_{k}(s, x)\right|^{p} d s\right) d x .
\end{aligned}
$$

Here, $u(t, x)=\left(u_{1}(t, x), u_{2}(t, x), \ldots, u_{n}(t, x)\right)^{T}$ is a solution for stochastic fuzzy system (8). Below, we may denote $u(t, x)$ by $u$ and $u_{i}(t, x)$ by $u_{i}$ for simplicity.

Remark 7. It is obvious that our Lyapunov-Krasovskii functional is the positive definite form of $p$ powers, which is different from those of existing literature ([41-43]). For example, in [41], the model is also neural networks with discrete time delay and distributed delays:

$$
\begin{aligned}
& d x(t)=\left[-C_{i} x(t)+A_{i} f(y(t-\tau(t), i))\right. \\
& \left.+B_{i} \int_{t-\rho(t)}^{t} f(y(s), r(s))\right] d t+\sigma_{t} d w_{1}(t), \\
& d y(t)=\left[-\widetilde{C}_{i} y(t)+\widetilde{A}_{i} g(x(t-\widetilde{\tau}(t), i))\right. \\
& \left.+\widetilde{B}_{i} \int_{t-\widetilde{\rho}(t)}^{t} g(x(s), r(s))\right] d t+\widetilde{\sigma}_{t} d w_{2}(t) .
\end{aligned}
$$


In [42, Theorem 1], the corresponding Lyapunov-Krasovskii functional is as follows:

$$
\begin{aligned}
\widetilde{V}_{2}= & \int_{-\rho}^{0} d \theta \int_{t+\theta}^{t} f^{T}(y(s), r(s)) L f(y(s), r(s)) d s \\
& +\int_{-\tilde{\rho}}^{0} d \theta \int_{t+\theta}^{t} g^{T}(x(s), r(s)) \operatorname{Lg}(x(s), r(s)) d s,
\end{aligned}
$$

which is the positive definite form of 2 powers. And the conclusion of [42, Theorem 1] is the asymptotical stability in the mean square, which is also similar to that of our Theorem 6. However, by means of our Lyapunov-Krasovskii functional with the positive definite form of $p$ powers, we shall derive the asymptotical stability in the mean square for nonlinear $p$-Laplacian diffusion system (8).

Evaluating the time derivation of $V_{1}(t)$ along the trajectory of the fuzzy system (8), we can get by [38, Lemma 6] and Lemma 4

$$
\begin{aligned}
& V_{1}^{\prime}(t)=2 \int_{\Omega}\left[u^{T} P\left(\nabla \cdot\left(D(t, x, u) \circ \nabla_{p} u\right)\right)\right. \\
& \left.-u^{T} P A(u) B(u)\right] d x+2 \sum_{j=1}^{r} h_{j}(\omega(t)) \\
& \quad \cdot\left(\int_{\Omega} u^{T} P A(u) c_{0} C_{j} f\left(u\left(t-\tau_{1}(t), x\right)\right) d x\right.
\end{aligned}
$$

$$
\begin{aligned}
c_{0} \int_{\Omega}\left|u^{T}\right| P A(u)\left|C_{j}\right|\left|f\left(u\left(t-\tau_{1}(t), x\right)\right)\right| d x & =c_{0} \sum_{k=1}^{n} \sum_{i=1}^{n} \int_{\Omega} p_{i}\left|u_{i} a_{i}\left(u_{i}\right)\right|\left|c_{i k}^{(j)}\right|\left|f_{k}\left(u_{k}\left(t-\tau_{1}(t), x\right)\right)\right| d x \\
& \leqslant c_{0} \bar{p} \sum_{k=1}^{n} \sum_{i=1}^{n} \int_{\Omega} \bar{a}_{i}\left|c_{j}\right|\left|u_{i}\right|^{p-1} F_{k}\left|u_{k}\left(t-\tau_{1}(t), x\right)\right| d x \\
& \leqslant c_{0} \bar{p}\left|c_{j}\right| \lambda_{\max } \bar{A} \lambda_{\max } F \sum_{k=1}^{n} \sum_{i=1}^{n} \int_{\Omega}\left(\frac{p-1}{p}\left|u_{i}\right|^{p}+\frac{\left|u_{k}\left(t-\tau_{1}(t), x\right)\right|^{p}}{p}\right) d x \\
& =c_{0} \frac{(p-1) \bar{p}}{p} n\left|c_{j}\right| \lambda_{\max } \bar{A} \lambda_{\max } F\|u\|_{p}^{p} \\
& +c_{0} \frac{\bar{p}}{p} n\left|c_{j}\right| \lambda_{\max } \bar{A} \lambda_{\max } F \sum_{k=1}^{n} \int_{\Omega}\left|u_{k}\left(t-\tau_{1}(t), x\right)\right|^{p} d x
\end{aligned}
$$

Besides, gathering ( $\mathrm{H} 1)$ and $(\mathrm{H} 2)$ gives

$$
\int_{\Omega} u^{T} P A(u) B(u) d x \geqslant \underline{p} \lambda_{\min }(\underline{A} \mathbb{B})\|u\|_{p}^{p} .
$$

It follows by (H1), (H3), and Lemma 3 that

Similarly,

$$
\begin{aligned}
& \left(1-c_{0}\right) \int_{\Omega}\left|u^{T}\right| P A(u)\left|C_{j}\right|\left|f\left(u\left(t-\tau_{2}(t), x\right)\right)\right| d x \leqslant\left(1-c_{0}\right) \frac{(p-1) \bar{p}}{p} n\left|c_{j}\right| \lambda_{\max } \bar{A} \lambda_{\max } F\|u\|_{p}^{p} \\
& \quad+\left(1-c_{0}\right) \frac{\bar{p}}{p} n\left|c_{j}\right| \lambda_{\max } \bar{A} \lambda_{\max } F \sum_{k=1}^{n} \int_{\Omega}\left|u_{k}\left(t-\tau_{2}(t), x\right)\right|^{p} d x,
\end{aligned}
$$




$$
\begin{aligned}
& \int_{\Omega}\left|u^{T}\right| P A(u)\left|M_{j}\right| \int_{t-\rho(t)}^{t}|f(u(s, x))| d s d x=\sum_{k=1}^{n} \sum_{i=1}^{n} \int_{\Omega} p_{i}\left|u_{i} a_{i}\left(u_{i}\right)\right|\left|m_{i k}^{(j)}\right| \int_{t-\rho(t)}^{t}\left|f_{k}\left(u_{k}(s, x)\right)\right| d s d x \\
& \leqslant \bar{p} \sum_{k=1}^{n} \sum_{i=1}^{n} \int_{\Omega} \int_{t-\rho(t)}^{t} \bar{a}_{i}\left|m_{j}\right|\left|u_{i}(t, x)\right|^{p-1} F_{k}\left|u_{k}(s, x)\right| d s d x \\
& \leqslant \bar{p}\left|m_{j}\right| \lambda_{\max } \bar{A} \lambda_{\max } F \sum_{k=1}^{n} \sum_{i=1}^{n} \int_{\Omega} \int_{t-\rho(t)}^{t}\left(\frac{p-1}{p}\left|u_{i}(t, x)\right|^{p}+\frac{\left|u_{k}(s, x)\right|^{p}}{p}\right) d s d x \leqslant \rho \frac{(p-1) \bar{p}}{p} n\left|m_{j}\right| \lambda_{\max } \bar{A} \lambda_{\max } F\|u\|_{p}^{p} \\
& +\frac{\bar{p}}{p}\left|m_{j}\right| \lambda_{\max } \bar{A} \lambda_{\max } F \sum_{i=1}^{n} \sum_{k=1}^{n} \int_{\Omega} \int_{t-\rho(t)}^{t}\left|u_{k}(s, x)\right|^{p} d s d x .
\end{aligned}
$$

On the other hand,

$$
\begin{aligned}
& V_{2}^{\prime}(t)=2 c_{0} n \frac{\bar{p}}{p}\left(\sum_{j=1}^{r}\left|c_{j}\right|\right) \\
& \cdot \lambda_{\max } \bar{A} \lambda_{\max } F\left(\sum_{k=1}^{n} \int_{\Omega}\left|u_{k}(t, x)\right|^{p} d x\right. \\
& \left.-\sum_{k=1}^{n}\left(1-\tau_{1}^{\prime}(t)\right) \int_{\Omega}\left|u_{k}\left(t-\tau_{1}(t), x\right)\right|^{p} d x\right) \\
& \leqslant 2 c_{0} n \frac{\bar{p}}{p}\left(\sum_{j=1}^{r}\left|c_{j}\right|\right) \lambda_{\max } \bar{A} \lambda_{\max } F\|u\|_{p}^{p}-2 c_{0} n \\
& \quad \frac{\bar{p}}{p}\left(\sum_{j=1}^{r}\left|c_{j}\right|\right) \lambda_{\max } \bar{A} \lambda_{\max } F\left(1-\tau_{1}\right) \\
& \quad \sum_{k=1}^{n} \int_{\Omega}\left|u_{k}\left(t-\tau_{1}(t), x\right)\right|^{p} d x .
\end{aligned}
$$

Similarly,

$$
\begin{aligned}
V_{3}^{\prime}(t) \leqslant & 2\left(1-c_{0}\right) n \frac{\bar{p}}{p}\left(\sum_{j=1}^{r}\left|c_{j}\right|\right) \lambda_{\max } \bar{A} \lambda_{\max } F\|u\|_{p}^{p} \\
& -2\left(1-c_{0}\right) n \frac{\bar{p}}{p}\left(\sum_{j=1}^{r}\left|c_{j}\right|\right) \\
& \cdot \lambda_{\max } \bar{A} \lambda_{\max } F\left(1-\tau_{2}\right) \\
& \cdot \sum_{k=1}^{n} \int_{\Omega}\left|u_{k}\left(t-\tau_{2}(t), x\right)\right|^{p} d x .
\end{aligned}
$$

Next, we need to recall some facts derived by mathematical analysis. Assume that $\eta(t, s)$ is continuous on variables $t$ and $s$, and $\partial \eta / \partial t$ exists, utilizing the integral middle value theorem reaches

$$
\begin{aligned}
\frac{d}{d t} \int_{\xi(t)}^{\omega(t)} \eta(t, s) d s= & \omega^{\prime}(t) \eta(t, \omega(t)) \\
& -\xi^{\prime}(t) \eta(t, \xi(t)) \\
& +\int_{\xi(t)}^{\omega(t)} \frac{\partial \eta(t, s)}{\partial t} d s
\end{aligned}
$$

where both $\xi(\cdot)$ and $\omega(\cdot)$ are differentiable.

Moreover, we can derive by employing (32) time and again

$$
\begin{aligned}
V_{4}^{\prime}(t) & =2 \frac{\bar{p}}{p}\left(\sum_{j=1}^{r}\left|m_{j}\right|\right) \lambda_{\max } \bar{A} \lambda_{\max } F \sum_{i=1}^{n} \sum_{k=1}^{n} \int_{\Omega}\left(\int_{-\rho}^{0}\left|u_{k}(t, x)\right|^{p} d s-\int_{-\rho}^{0}\left|u_{k}(t+s, x)\right|^{p} d s\right) d x \\
& =2 \frac{\bar{p}}{p}\left(\sum_{j=1}^{r}\left|m_{j}\right|\right) \lambda_{\max } \bar{A} \lambda_{\max } F \sum_{i=1}^{n} \sum_{k=1}^{n} \int_{\Omega}\left(\rho\left|u_{k}(t, x)\right|^{p}-\int_{t-\rho(t)}^{t}\left|u_{k}(s, x)\right|^{p} d s\right) d x \\
& =2 \frac{\bar{p}}{p}\left(\sum_{j=1}^{r}\left|m_{j}\right|\right) \lambda_{\max } \bar{A} \lambda_{\max } F\left(n \rho\|u\|_{p}^{p}-\sum_{i=1}^{n} \sum_{k=1}^{n} \int_{\Omega} \int_{t-\rho(t)}^{t}\left|u_{k}(s, x)\right|^{p} d s d x\right) .
\end{aligned}
$$


Combining (28)-(35) results in

$$
\begin{aligned}
& V^{\prime}(t) \leqslant-2\left[\lambda_{1} D \underline{p}+\underline{p} \lambda_{\min }(\underline{A} \mathbb{B})-\frac{n}{p}\right. \\
& \cdot \sum_{j=1}^{r}\left(\bar{p}(p-1)\left|c_{j}\right|+\rho(p-1) \bar{p}\left|m_{j}\right|\right. \\
& \left.+c_{0} \frac{\bar{p}}{1-\tau_{1}}\left|c_{j}\right|+\left(1-c_{0}\right) \frac{\bar{p}}{1-\tau_{2}}\left|c_{j}\right|+\rho \bar{p}\left|m_{j}\right|\right) \\
& \left.\cdot \lambda_{\max } \bar{A} \lambda_{\max } F\right]\|u\|_{p}^{p} \leqslant 0 .
\end{aligned}
$$

Now the standard Lyapunov functional theory derives that the null solution of the fuzzy system (8) is globally asymptotically stable.

Remark 8. In the case of Takagi-Sugeno fuzzy model, our Theorem 6 is better than [38, Theorem 4] because the condition (H1) is weaker than the bounded assumption (2).

Remark 9. In Theorem 6, (22) illustrates the influence of nonlinear diffusion on the stability of system (8) while its role was always ignored in existing results (see, e.g., $[5,39,44]$ ).

Theorem 6 derives the following corollary.

Corollary 10. If there exists a positive definite matrix $P=$ $\operatorname{diag}\left(p_{1}, p_{2}, \ldots, p_{n}\right)$ and two positive scalars $p, \bar{p}$ such that the following inequalities hold:

$$
\begin{aligned}
& \underline{p} \lambda_{\text {min }}(\underline{A} \mathbb{B})>\frac{n \bar{p}}{p} \sum_{j=1}^{r}\left((p-1)\left|c_{j}\right|+\rho(p-1)\left|m_{j}\right|\right. \\
& \left.\quad+\frac{c_{0}\left|c_{j}\right|}{1-\tau_{1}}+\frac{\left(1-c_{0}\right)\left|c_{j}\right|}{1-\tau_{2}}+\rho\left|m_{j}\right|\right) \lambda_{\text {max }} \bar{A} \lambda_{\text {max }} F, \\
& P>\underline{p} I, \\
& P<\bar{p} I,
\end{aligned}
$$

then the null solution of the ordinary integrodifferential equations (9) is globally asymptotically stable.

Furthermore, if both diffusion behaviors and distributed delay are ignored, we derive from Corollary 10.

Corollary 11. If there exists a positive definite matrix $P=$ $\operatorname{diag}\left(p_{1}, p_{2}, \ldots, p_{n}\right)$ and two positive scalars $p, \bar{p}$ such that the following inequalities hold:

$$
\begin{aligned}
& \underline{p} \lambda_{\min }(\underline{A} \mathbb{B})>\frac{n \bar{p}}{p} \\
& \quad \cdot \sum_{j=1}^{r}\left((p-1)\left|c_{j}\right|+\frac{c_{0}\left|c_{j}\right|}{1-\tau_{1}}+\frac{\left(1-c_{0}\right)\left|c_{j}\right|}{1-\tau_{2}}\right)
\end{aligned}
$$

$$
\begin{aligned}
& \cdot \lambda_{\max } \bar{A} \lambda_{\max } F, \\
P & >\underline{p} I, \\
P & <\bar{p} I,
\end{aligned}
$$

then the null solution of the following fuzzy system

$$
\begin{aligned}
& \frac{d x(t)}{d t}=-A(x(t))\left\{B(x(t))-\sum_{j=1}^{r} h_{j}(\omega(t))\right. \\
& \cdot\left[c_{0} C_{j} f\left(x\left(t-\tau_{1}(t)\right)\right)+\left(1-c_{0}\right)\right. \\
& \cdot C_{j} f\left(x\left(t-\tau_{2}(t)\right)\right)+\left(\mathfrak{c}-c_{0}\right) \\
& \left.\left.\cdot\left(C_{j} f\left(x\left(t-\tau_{1}(t)\right)\right)-C_{j} f\left(x\left(t-\tau_{2}(t)\right)\right)\right)\right]\right\}, \\
& x(\theta)=\phi(\theta), \quad \theta \in(-\infty, 0]
\end{aligned}
$$

is globally asymptotically stable.

Remark 12. Condition (H1) is weaker than the bounded conditions on amplification functions of existing literature $([3-6,9,10,24,27,28])$.

Discussion 1. In recent related literature ([27, 45-51]), some new conditions and methods were presented, and their results were very good. However, some of the methods and conditions are not applicable for system (8) with nonlinear $p$-Laplacian diffusion. How to apply the new conditions and methods of [45-49] to our system (8) is an interesting problem.

\section{Methods and Numerical Example}

4.1. Methods. In this paper, Lyapunov functional method is employed to derive the stability criterion. In this process, the integral middle value theorem together with the derivation formula on integral upper limit functions plays the important roles.

Example 1. Consider the following Takagi-Sugeno $p$-Laplace fuzzy T-S dynamic equations.

Fuzzy Rule 1. IF $\omega_{1}(t)$ is $\mu_{11}$, and $\omega_{2}(t)$ is $\mu_{12}$, THEN

$$
\begin{aligned}
\frac{\partial u}{\partial t} & =\nabla \cdot\left(\mathscr{D}(t, x, u) \circ \nabla_{p} u\right)-A(u)[B(u) \\
& -c_{0} C_{1} f\left(u\left(t-\tau_{1}(t), x\right)\right)-\left(1-c_{0}\right) \\
& \cdot C_{1} f\left(u\left(t-\tau_{2}(t), x\right)\right)-\left(\mathfrak{s}-c_{0}\right) \\
& \cdot\left(C_{1} f\left(u\left(t-\tau_{1}(t), x\right)\right)-C_{1} f\left(u\left(t-\tau_{2}(t), x\right)\right)\right) \\
& \left.-M_{1} \int_{t-\rho(t)}^{t} f(u(s, x)) d s\right],
\end{aligned}
$$




$$
\begin{aligned}
& u(\theta, x)=\phi(\theta, x), \quad(\theta, x) \in(-\infty, 0] \times \Omega, \\
& u(t, x)=0 \in R^{2}, \quad(t, x) \in R \times \partial \Omega .
\end{aligned}
$$

Fuzzy Rule 2. IF $\omega_{1}(t)$ is $\mu_{21}$, and $\omega_{2}(t)$ is $\mu_{22}$, THEN

$$
\begin{aligned}
& \frac{\partial u}{\partial t}=\nabla \cdot\left(\mathscr{D}(t, x, u) \circ \nabla_{p} u\right)-A(u)[B(u) \\
& -c_{0} C_{2} f\left(u\left(t-\tau_{1}(t), x\right)\right)-\left(1-c_{0}\right) \\
& \cdot C_{2} f\left(u\left(t-\tau_{2}(t), x\right)\right)-\left(\mathfrak{c}-c_{0}\right) \\
& \cdot\left(C_{2} f\left(u\left(t-\tau_{1}(t), x\right)\right)-C_{2} f\left(u\left(t-\tau_{2}(t), x\right)\right)\right) \\
& \left.-M_{2} \int_{t-\rho(t)}^{t} f(u(s, x)) d s\right] \\
& u(\theta, x)=\phi(\theta, x), \quad(\theta, x) \in(-\infty, 0] \times \Omega, \\
& u(t, x)=0 \in R^{2}, \quad(t, x) \in R \times \partial \Omega,
\end{aligned}
$$

where $u(t, x)=\left(u_{1}(t, x), u_{2}(t, x)\right)^{T}, \Omega=(0, \pi), p=4 / 3$, and then Remark 1 gives

$$
\lambda_{1}=\left(\frac{2}{\pi} \int_{0}^{(p-1)^{1 / p}} \frac{d t}{\left(1-t^{p} /(p-1)\right)^{1 / p}}\right)^{p}=0.7915
$$

Let $\tau_{1}(t)=t / 3, \tau_{2}(t)=t / 2$, and then $\tau_{1}=1 / 3, \tau_{2}=$ 1/2. Let $a_{i}\left(u_{i}\right)=0.1 u_{i}^{-2 / 3}\left(1+e^{-i u_{i}^{2}}\right), i=1,2, b_{1}\left(u_{1}\right)=$ $2 u_{1}\left(1+\sin ^{2} u_{1}\right), b_{2}\left(u_{2}\right)=1.95 u_{2}, f_{1}\left(u_{1}(t-\tau(t))\right)=0.16 u_{1}(t-$ $\tau(t)) \sin u_{1}(t-\tau(t)), f_{2}\left(u_{2}(t-\tau(t))\right)=0.166 u_{2}(t-\tau(t)), \tau=$ $0.5, D=0.003, c_{0}=0.75, c_{1}=0.2, c_{2}=0.3, m_{1}=$ $0.02, m_{2}=0.03$, and

$$
\begin{aligned}
\underline{A} & =\left(\begin{array}{cc}
0.01 & 0 \\
0 & 0.02
\end{array}\right), \\
\bar{A} & =\left(\begin{array}{cc}
0.1 & 0 \\
0 & 0.2
\end{array}\right), \\
\mathbb{B} & =\left(\begin{array}{cc}
2 & 0 \\
0 & 1.95
\end{array}\right), \\
D(t, x, u) & =\left(\begin{array}{cc}
0.003 & 0.005 \\
0.004 & 0.006
\end{array}\right), \\
M_{1} & =\left(\begin{array}{cc}
0.02 & 0.01 \\
0 & 0.01
\end{array}\right),
\end{aligned}
$$

$$
F=\left(\begin{array}{cc}
0.16 & 0 \\
0 & 0.166
\end{array}\right)
$$$$
C_{1}=\left(\begin{array}{cc}
0.2 & 0.1 \\
0 & 0.15
\end{array}\right)
$$$$
C_{2}=\left(\begin{array}{cc}
0.2 & 0.1 \\
0 & 0.3
\end{array}\right)
$$$$
M_{2}=\left(\begin{array}{cc}
0.01 & 0.01 \\
0 & 0.03
\end{array}\right)
$$

Now we use MATLAB to solve LMIs (22)-(23), obtaining the feasibility data

$$
\begin{aligned}
& P=\left(\begin{array}{cc}
0.9381 & 0 \\
0 & 1.013
\end{array}\right), \\
& \underline{p}=0.9103, \\
& \bar{p}=1.023 .
\end{aligned}
$$

Now Theorem 6 derives that the null solution of this Takagi-Sugeno fuzzy equations is globally asymptotically stable (see Figures 1 and 2).

\section{Conclusions}

By constructing a novel Lyapunov function, we employed Young inequality and LMI technique to derive the asymptotic stability criteria for CGNNs with distributed delays and nonlinear diffusion. Since the stability of nonlinear $p$-Laplacian diffusion neural networks was originally investigated in [2], various $p$-Laplacian diffusion neural networks have attracted a lot of interest $([6,17,34,39,44])$. As pointed out in Discussion 1, some new conditions and methods may not be applicable to CGNNs model with nonlinear $p$-Laplacian diffusion. So our results are a novelty to some extent.

\section{Conflicts of Interest}

The authors declare that there are no conflicts of interest regarding the publication of this paper.

\section{Authors' Contributions}

Xiongrui Wang wrote the original manuscript, carrying out the main part of this paper. Shouming Zhong checked it, and Ruofeng Rao is in charge of correspondence. All authors read and approved the final manuscript.

\section{Acknowledgments}

This work is supported by Scientific Research Fund of Science Technology Department of Sichuan Province (2011)YZ010, 

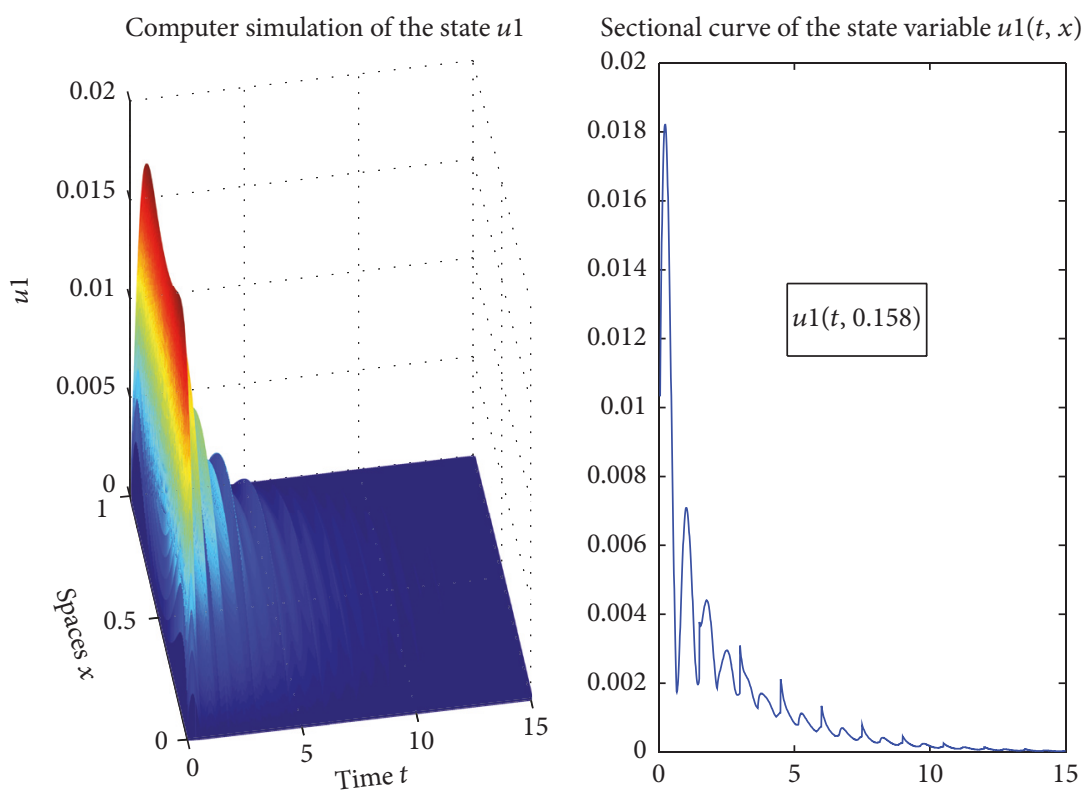

FIgURE 1: Computer simulations of the state $u_{1}(t, x)$.
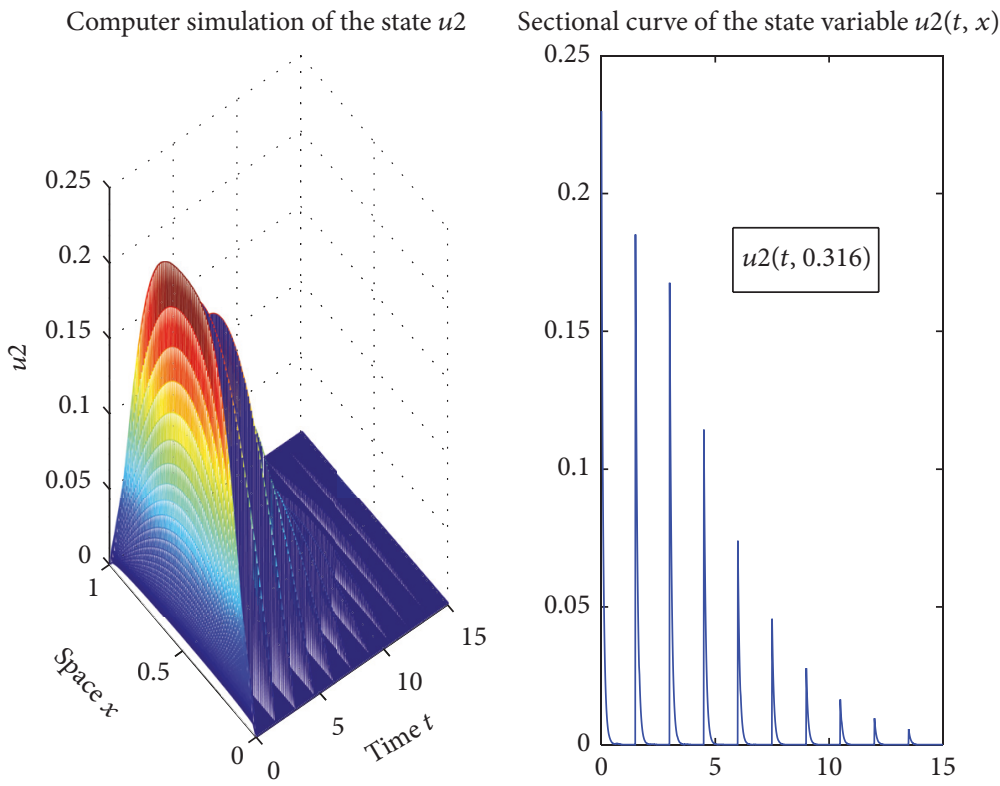

Figure 2: Computer simulations of the state $u_{2}(t, x)$.

2012JY010) and Scientific Research Fund of Sichuan Provincial Education Department (14ZA0274, 12ZB349, 11ZA172, and 08ZB002).

\section{References}

[1] X. Zhang, S. Wu, and K. Li, "Delay-dependent exponential stability for impulsive Cohen-Grossberg neural networks with time-varying delays and reaction-diffusion terms," Communications in Nonlinear Science and Numerical Simulation, vol. 16, no. 3, pp. 1524-1532, 2011.
[2] R. Rao, S. Zhong, and X. Wang, "Stochastic stability criteria with LMI conditions for Markovian jumping impulsive BAM neural networks with mode-dependent time-varying delays and nonlinear reaction-diffusion," Communications in Nonlinear Science and Numerical Simulation, vol. 19, no. 1, pp. 258-273, 2014.

[3] Q. Liu and R. Xu, "Periodic solutions of a cohen-grossberg-type BAM neural networks with distributed delays and impulses," Journal of Applied Mathematics, vol. 2012, Article ID 643418, 17 pages, 2012.

[4] H. Song, D. Chen, W. Li, and Y. Qu, "Graph-theoretic approach to exponential synchronization of stochastic reaction-diffusion 
Cohen-Grossberg neural networks with time-varying delays," Neurocomputing, vol. 177, pp. 179-187, 2016.

[5] H. Xiang and J. Cao, "Periodic oscillation of fuzzy cohengrossberg neural networks with distributed delay and variable coefficients," Journal of Applied Mathematics, vol. 2008, Article ID 453627, 18 pages, 2008.

[6] P. Qingfei, Z. Zifang, and H. Jingchang, "Stability of the stochastic reaction-diffusion neural network with time-varying delays and p-laplacian," Journal of Applied Mathematics, vol. 2012, Article ID 405939, 10 pages, 2012.

[7] Q. Song, H. Yan, Z. Zhao, and Y. Liu, "Global exponential stability of impulsive complex-valued neural networks with both asynchronous time-varying and continuously distributed delays," Neural Networks, vol. 81, pp. 1-10, 2016.

[8] K. Li, L. Zhang, X. Zhang, and Z. Li, "Stability in impulsive Cohen-Grossberg-type BAM neural networks with distributed delays," Applied Mathematics and Computation, vol. 215, no. 11, pp. 3970-3984, 2010.

[9] K. Li and Q. Song, "Exponential stability of impulsive CohenGrossberg neural networks with time-varying delays and reaction-diffusion terms," Neurocomputing, vol. 72, no. 1-3, pp. 231-240, 2008.

[10] J. Pan and S. Zhong, "Dynamic analysis of stochastic reactiondiffusion Cohen-Grossberg neural networks with delays," Advances in Difference Equations, vol. 2009, Article ID 410823, pp. 1-18, 2009.

[11] R. Rao, X. Wang, and S. Zhong, "LMI-based stability criterion for impulsive delays Markovian jumping time-delays reactiondiffusion BAM neural networks via Gronwall-Bellman-type impulsive integral inequality," Mathematical Problems in Engineering, vol. 2015, Article ID 185854, 11 pages, 2015.

[12] Q. Song, J. Cao, and Z. Zhao, "Periodic solutions and its exponential stability of reaction-diffusion recurrent neural networks with continuously distributed delays," Nonlinear Analysis: Real World Applications, vol. 7, no. 1, pp. 65-80, 2006.

[13] R. Rao, Z. Pu, S. Zhong, and X. Li, "Fixed points and exponential stability for impulsive time-delays BAM neural networks via LMI approach and contraction mapping principle," Mathematical Problems in Engineering, vol. 2016, Article ID 3154683, 8 pages, 2016.

[14] Y. Li, Y. Mi, and C. Mu, "Properties of positive solutions for a nonlocal nonlinear diffusion equation with nonlocal nonlinear boundary condition," Acta Mathematica Scientia, vol. 34, no. 3, pp. 748-758, 2014.

[15] R. Rao and S. Zhong, "Stability analysis of impulsive stochastic reaction-diffusion cellular neural network with distributed delay via fixed point theory," Complexity, Article ID 6292597, 9 pages, 2017.

[16] V. A. Galaktionov, "On asymptotic self-similar behaviour for a quasilinear heat equation: single point blow-up," SIAM Journal on Mathematical Analysis, vol. 26, no. 3, pp. 675-693, 1995.

[17] R. Rao and S. Zhong, "Existence of exponential p-stability nonconstant equilibrium of markovian jumping nonlinear diffusion equations via ekeland variational principle," Advances in Mathematical Physics, vol. 2015, Article ID 812150, 10 pages, 2015.

[18] R. Rao, S. Zhong, and Z. Pu, "On the role of diffusion factors in stability analysis for p-Laplace dynamical equations involved to BAM Cohen-Grossberg neural network," Neurocomputing, vol. 223, pp. 54-62, 2017.

[19] J. Ding and B.-Z. Guo, "Global existence and blow-up solutions for quasilinear reactiondiffusion equations with a gradient term," Applied Mathematics Letters, vol. 24, no. 6, pp. 936-942, 2011.

[20] R. Rao and Z. Pu, "Stability analysis for impulsive stochastic fuzzy p-Laplace dynamic equations under Neumann or Dirichlet boundary condition," Boundary Value Problems, vol. 2013, article no. 133, 2013.

[21] Y. H. Xia, Z. Yang, and M. Han, "Lag synchronization of unknown chaotic delayed yang-yang-type fuzzy neural networks with noise perturbation based on adaptive control and parameter identification," IEEE Transactions on Neural Networks and Learning Systems, vol. 20, no. 7, pp. 1165-1180, 2009.

[22] Y. Xia, Z. Yang, and M. Han, "Synchronization schemes for coupled identical YANg-YANg type fuzzy cellular neural networks," Communications in Nonlinear Science and Numerical Simulation, vol. 14, no. 9-10, pp. 3645-3659, 2009.

[23] D. He and D. Xu, "Attracting and invariant sets of fuzzy CohenGrossberg neural networks with time-varying delays," Physics Letters A, vol. 372, no. 47, pp. 7057-7062, 2008.

[24] Y. Liu and W. Tang, "Exponential stability of fuzzy cellular neural networks with constant and time-varying delays," Physics Letters A, vol. 323, no. 3-4, pp. 224-233, 2004.

[25] C. K. Ahn, "Delay-dependent state estimation for T-S fuzzy delayed Hopfield neural networks," Nonlinear Dynamics, vol. 61, no. 3, pp. 483-489, 2010.

[26] Q. Song and J. Cao, "Impulsive effects on stability of fuzzy Cohen-Grossberg neural networks with time-varying delays," IEEE Transactions on Systems, Man, and Cybernetics, Part B: Cybernetics, vol. 37, no. 3, pp. 733-741, 2007.

[27] Q. Zhu and X. Li, "Exponential and almost sure exponential stability of stochastic fuzzy delayed Cohen-Grossberg neural networks," Fuzzy Sets and Systems, vol. 203, pp. 74-94, 2012.

[28] M. A. Cohen and S. Grossberg, "Absolute stability of global pattern formation and parallel memory storage by competitive neural networks," IEEE Transactions on Systems, Man, and Cybernetics, vol. 13, no. 5, pp. 815-826, 1983.

[29] A.-T. Nguyen, T. Laurain, R. Palhares, J. Lauber, C. Sentouh, and J.-C. Popieul, "LMI-based control synthesis of constrained Takagi-Sugeno fuzzy systems subject to L2 or Lo disturbances," Neurocomputing, vol. 207, pp. 793-804, 2016.

[30] R. Mrquez, T. M. Guerra, M. Bernal, and A. Kruszewski, "Asymptotically necessary and sufficient conditions for Takagi-Sugeno models using generalized non-quadratic parameter-dependent controller design," Fuzzy Sets and Systems, vol. 306, pp. 48-62, 2017.

[31] S. Xu, G. Sun, and W. Sun, “Takagi-Sugeno fuzzy model based robust dissipative control for uncertain flexible spacecraft with saturated time-delay input," ISA Transactions, vol. 66, pp. 105121, 2017.

[32] X. M. Ding, Z. K. Xu, N. J. Cheung, and X. H. Liu, "Parameter estimation of Takagi-Sugeno fuzzy system using heterogeneous cuckoo search algorithm," Neurocomputing, vol. 151, no. 3, pp. 1332-1342, 2015.

[33] H. D. Choi, C. K. Ahn, P. Shi, M. T. Lim, and M. K. Song, "L2Lo Filtering for takagi-sugeno fuzzy neural networks based on wirtinger-type inequalities," Neurocomputing, vol. 153, pp. 117125, 2015.

[34] X. Wang, R. Rao, and S. Zhong, "LMI approach to stability analysis of Cohen-Grossberg neural networks with p-Laplace diffusion," Journal of Applied Mathematics, vol. 2012, Article ID 523812, 12 pages, 2012. 
[35] X. Li, R. Rakkiyappan, and P. Balasubramaniam, "Existence and global stability analysis of equilibrium of fuzzy cellular neural networks with time delay in the leakage term under impulsive perturbations," Journal of The Franklin Institute, vol. 348, no. 2, pp. 135-155, 2011.

[36] S. Muralisankar and N. Gopalakrishnan, "Robust stability criteria for Takagi-Sugeno fuzzy Cohen-Grossberg neural networks of neutral type," Neurocomputing, vol. 144, pp. 516-525, 2014.

[37] P. Balasubramaniam and M. Syed Ali, "Stability analysis of Takagi-Sugeno fuzzy Cohen-Grossberg BAM neural networks with discrete and distributed time-varying delays," Mathematical and Computer Modelling, vol. 53, no. 1-2, pp. 151-160, 2011.

[38] P. Lindqvist, "On the equation $\operatorname{div}\left(|\nabla u|^{p-2}\right)+\lambda|u|^{p-2} u=0$," Proceedings of the American Mathematical Society, vol. 109, no. 1, pp. 159-164, 1990.

[39] R. Rao, "Delay-dependent exponential stability for nonlinear reaction-diffusion uncertain Cohen-Grossberg neural networks with partially known transition rates via Hardy-Poincare inequality," Chinese Annals of Mathematics, Series B, vol. 35, no. 4, pp. 575-598, 2014.

[40] R. F. Rao and X. R. Wang, "Infinitely many solutions for the resonant quasi-linear equation without landesman-lazer conditions," Acta Mathematica Scientia (Chinese Series), vol. 32, no. 4, pp. 744-752, 2012.

[41] Q. Zhu, C. Huang, and X. Yang, "Exponential stability for stochastic jumping BAM neural networks with time-varying and distributed delays," Nonlinear Analysis: Hybrid Systems, vol. 5, no. 1, pp. 52-77, 2011.

[42] Z. Chen, X. Wang, S. Zhong, and J. Yang, "Improved delaydependent robust passivity criteria for uncertain neural networks with discrete and distributed delays," Chaos, Solitons \& Fractals, vol. 103, pp. 23-32, 2017.

[43] R. Yang and R. Guo, "Adaptive finite-time robust control of nonlinear delay hamiltonian systems via lyapunov-krasovskii method," Asian Journal of Control, vol. 20, no. 2, article 1C11, 2008.

[44] R. Rao, Z. Pu, S. Zhong, and J. Huang, "On the role of diffusion behaviors in stability criterion for p-laplace dynamical equations with infinite delay and partial fuzzy parameters under dirichlet boundary value," Journal of Applied Mathematics, vol. 2013, Article ID 940845, 8 pages, 2013.

[45] C. K. Ahn, "Passive and exponential filter design for fuzzy neural networks," Information Sciences, vol. 238, pp. 126-137, 2013.

[46] X. Li and J. Wu, "Stability of nonlinear differential systems with state-dependent delayed impulses," Automatica, vol. 64, pp. 6369, 2016.

[47] J. Cheng, H. Zhu, S. Zhong, Q. Zhong, and Y. Zeng, "Finite-time $H_{\infty}$ estimation for discrete-time Markov jump systems with time-varying transition probabilities subject to average dwell time switching," Communications in Nonlinear Science and Numerical Simulation, vol. 20, no. 2, pp. 571-582, 2015.

[48] Q. K. Song and Z. J. Zhao, "Stability criterion of complex-valued neural networks with both leakage delay and time-varying delays on time scales," Neurocomputing, vol. 171, pp. 179-184, 2016.

[49] X. Li and F. Deng, "Razumikhin method for impulsive functional differential equations of neutral type," Chaos, Solitons \& Fractals, vol. 101, pp. 41-49, 2017.

[50] J. Tian, W. Xiong, and F. Xu, "Improved delay-partitioning method to stability analysis for neural networks with discrete and distributed time-varying delays," Applied Mathematics and Computation, vol. 233, pp. 152-164, 2014.

[51] X. Li and J. Cao, "An impulsive delay inequality involving unbounded time-varying delay and applications," Institute of Electrical and Electronics Engineers Transactions on Automatic Control, vol. 62, no. 7, pp. 3618-3625, 2017. 


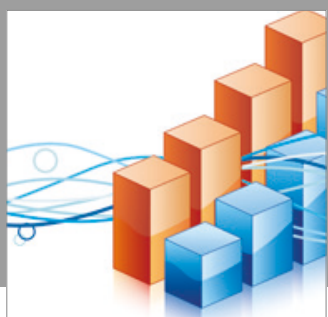

Advances in

Operations Research

vatersals

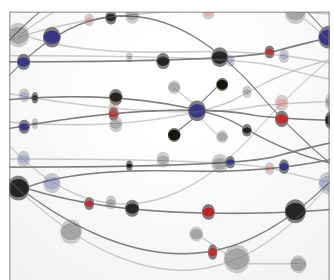

\section{The Scientific} World Journal
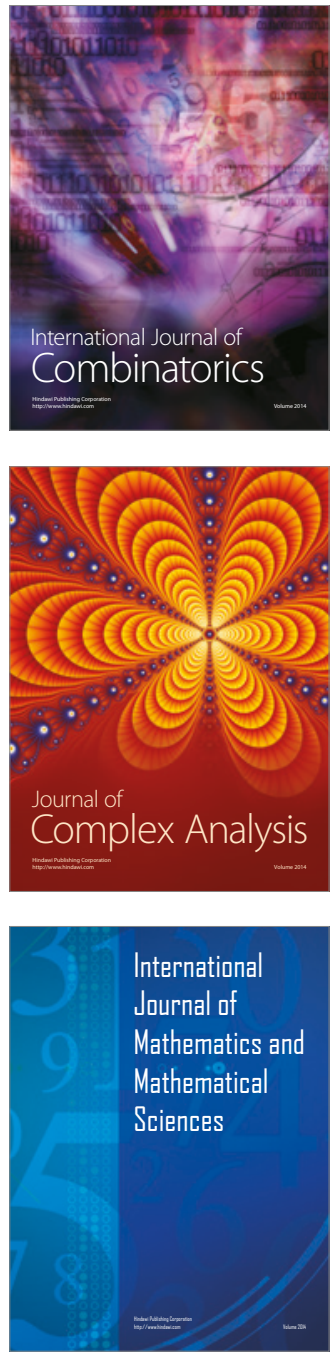
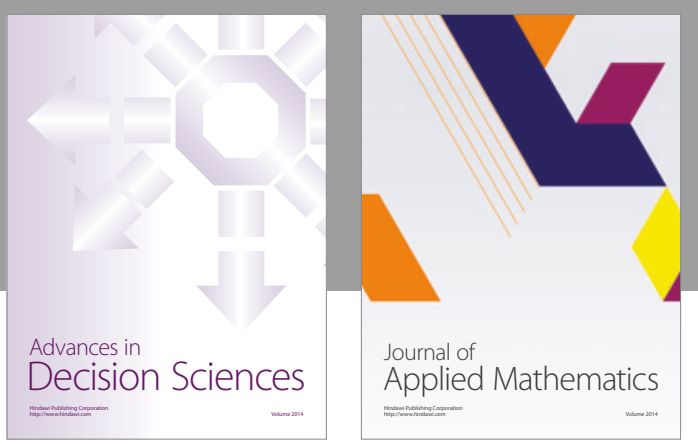

Algebra

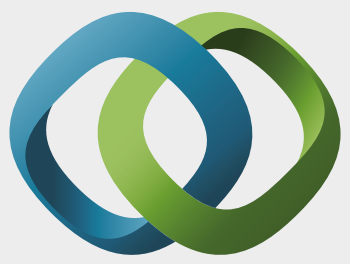

\section{Hindawi}

Submit your manuscripts at

https://www.hindawi.com
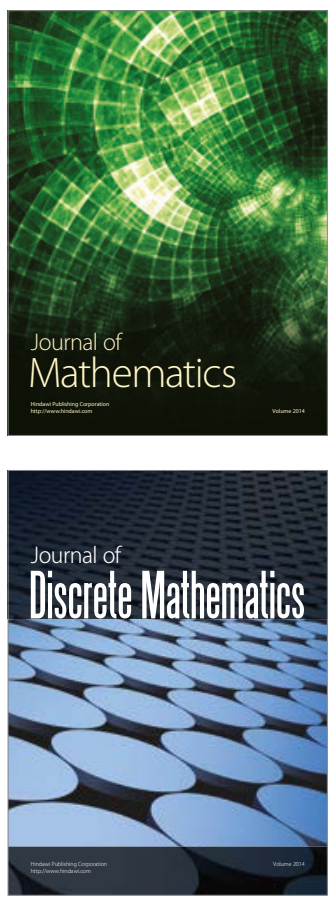

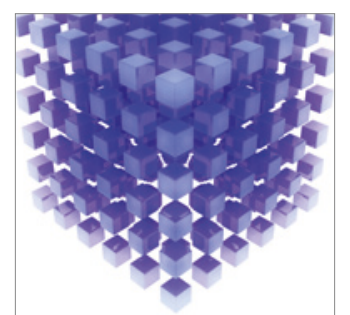

Mathematical Problems in Engineering
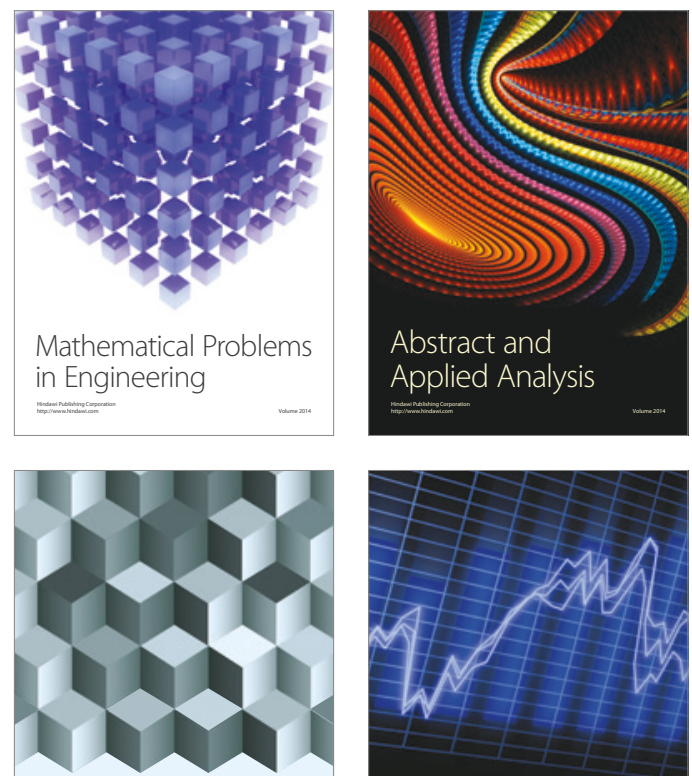

Journal of

Function Spaces

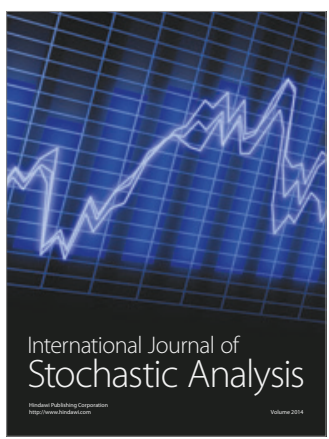

Probability and Statistics
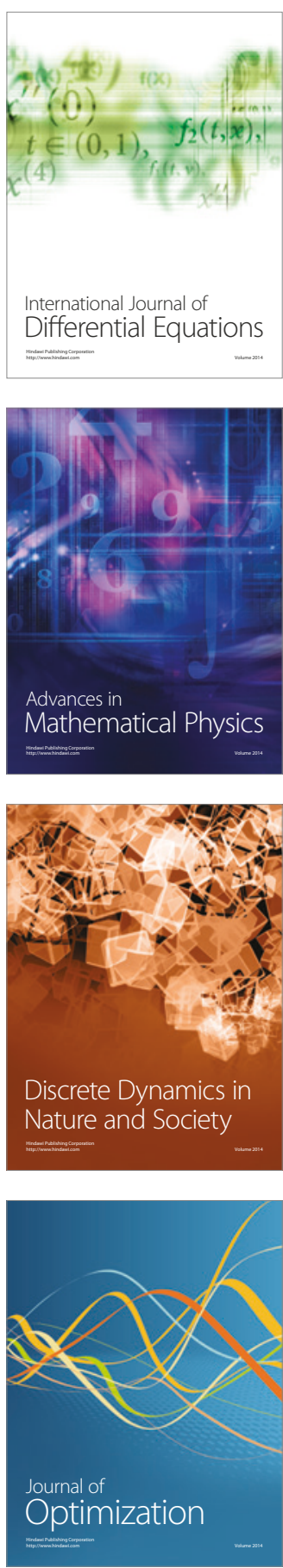